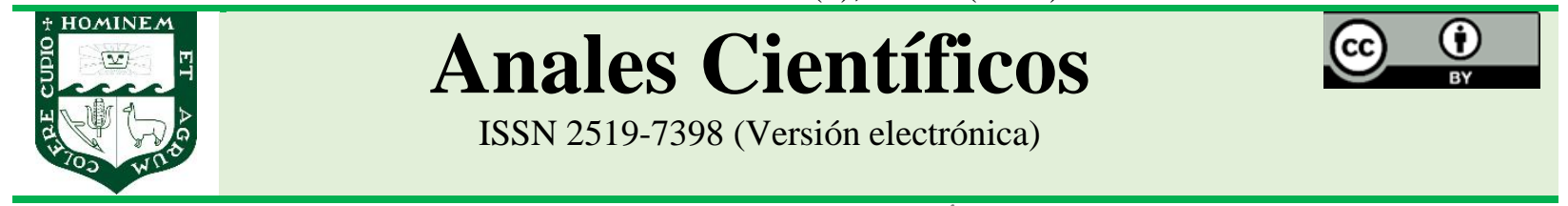

ARTÍCULO ORIGINAL - RESEARCH ARTICLE

http://dx.doi.org/10.21704/ac.v82i1.1741

\title{
INCIDENCIA COMERCIAL DE LA LEY DE PROMOCIÓN AGRARIA Y LA DEMANDA MUNDIAL EN LAS EXPORTACIONES ALIMENTOS PROCESADOS Y AGROALIMENTARIOS, CASO PERÚ: 2001-2018
}

\section{Commercial impact of agricultural promotion law and world demand in the exports of processed food and agro-based products, case Peru: 2001-2018}

\author{
Claudio Castagnino Pastor ${ }^{1}$; Luis Guillen Vidal ${ }^{1 *(1)}$ \\ ${ }^{1}$ Facultad de Economía y Planificación, Universidad Nacional Agraria La Molina, La Molina, 15024, Lima, Perú. \\ *E-mail: lguillen@lamolina.edu.pe
}

Recibido: 29/04/2020; Aceptado: 19/0272021; Publicado: 31/08/2021

\begin{abstract}
Faced with a context of global integration and growth of agricultural exports in Peru. The objective of the research was to examine the effected of the Agrarian Promotion Law (law 27360) and world demand during the period 20012018 on the export of the food group of processed food and agro-based products in Peru. The methodology evaluated using the Johansen co-integration test and the Granger causality test: world demand (world Imports) and exports of processed food and agro-based products from the countries of the Andean Community (CAN), where only two members (Peru and Ecuador) had laws to promote the agricultural. Our results showed that there is no causality between the exports of each member of the CAN and world demand in the food group, meanwhile, existed a long-term relationship between exports from Peru and Ecuador with world demand in the food group. The research concluded that Law 27360 and world demand of food group affected positive in the long-term exports of processed food and agro-based products from Peru.
\end{abstract}

Keywords: Bolivia; Colombia; Ecuador; Perú; Johansen Test; Granger Causality; Law 27360

\section{RESUMEN}

Ante un contexto de integración mundial e incremento de las exportaciones de alimentos procesados y agroalimentarios en Perú. La investigación tuvo el objetivo determinar el efecto de la Ley de Promoción Agraria (ley 27360) y la demanda mundial durante el 2001-2018 en el sector de la exportación de productos agroalimentarios y alimentos procesados en Perú. La metodología evaluó mediante la prueba de cointegración de Johansen y la prueba de causalidad de Granger: la demanda mundial (importaciones mundiales) y exportaciones de los países de la comunidad Andina (CAN) en el sector agroalimentarios y alimentos procesados, donde solo dos miembros (Perú y Ecuador) tenían leyes para promover o fomentar al sector en estudio. Los resultados mostraron que no hay causalidad entre las exportaciones de cada miembro de la CAN y la demanda mundial en el sector, mientras tanto, existía una relación a largo plazo entre las exportaciones de Perú y Ecuador con la demanda mundial en el sector. La investigación concluyó que la ley 27360 y la demanda de alimentos mundial afectaron positivamente las exportaciones a largo plazo de productos agroalimentarios y alimentos procesados del Perú. 


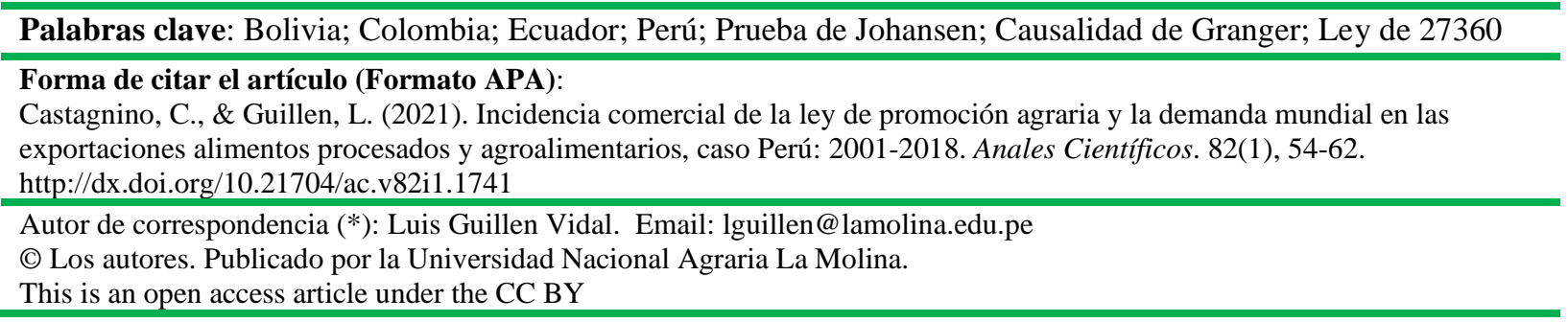

\section{INTRODUCCIÓN}

Las exportaciones alimentos procesados y productos agroalimentarios del Perú desde el 2000 hasta 2018 han aumentado considerablemente de acuerdo con el International Trade Centre en el 2019 (Anexo 3), dicho comportamiento pudo ser impulsado tanto por la Ley de Promoción Agraria (Ley 27360) como por la importación mundial del grupo productos agroalimentarios y alimentos procesados (demanda mundial de alimentos).

De acuerdo, a la Sociedad de Comercio Exterior del Perú (COMEX Perú, 2019) las agroexportaciones crecieron de 643 millones de US\$ en 2000 a 6651 millones de US\$ en 2018, el empleo formal creció de $16 \%$ (2004) a $25 \%$ (2017), las contribuciones al estado peruano durante el periodo del 2001 al 2017 incrementó de S/ 98 millones a S/ 539 millones. Superintendencia Nacional de Aduanas y de Administración Tributaria (SUNAT, 2019) reportó: el Perú exportó productos no tradicionales como: espárragos (frescos o refrigerados), uvas frescas, aguacates (paltas) frescas o secas, espárragos preparados o conservados, sin congelar.

Referente a las exportaciones del Perú, la Organización de las Naciones Unidas para la Agricultura y la Alimentación y Corporación Andina de Fomento (FAO-CAF, 2019) señaló respecto a los mercados destino en 2004: la Unión Europea y los Estados Unidos, representan los dos (02) grandes mercados para las exportaciones agrícolas, con $32 \%$ y $43 \%$ respectivamente. En 2019, según International Trade Center (ITC) sobre las exportaciones de Perú: China representó el 27\% de alimentos procesados y agroalimentarios en 2004 y $31 \%$ en 2018; Estados Unidos 12\% y España el $8 \%$ de las exportaciones de alimentos procesados y agroalimentarios en 2018.
En el proceso de apertura al mercado mundial, en 1995, Perú se incorporó al Tratado de Libre Comercio Mundial (Organización Mundial de Comercio, 2019), permitiendo un mejor acceso a la demanda mundial de productos agroalimentarios y alimentos procesados. En octubre del 2000 en el diario oficial El Peruano fue publicada la Ley 27360 con el objetivo de priorizar las inversiones y desarrollo agrario (El Peruano, 2000). Afirmando la apertura comercial, en el año 2013, se firma el Tratado de Libre Comercio entre Perú y la Unión Europea (León, 2013), entre otros tratados de Libre Comercio ampliando los mercados para los productos del Perú. Además, Perú cuenta con otros mecanismos normativos como: Ley de Promoción Comercial y Erradicación de la Droga, Programa de Renovación de Cultivos de Coca, este benefició a los países de la CAN (Perú, Ecuador, Colombia y Bolivia) siendo renovada en el 2001 después de 10 años de vigencia por los Estados Unidos, impulsando el comercio entre los Estados Unidos y los países de la CAN.

Las exportaciones pueden ayudar a un país a integrarse a la economía del mundo, así como a reducir el impacto de los golpes externos en la economía doméstica. Asimismo, las exportaciones permiten a la producción doméstica llegar a altos niveles de escala. La experiencia de los países del este asiático permite identificar a las exportaciones como un motor para el crecimiento económico (Jordaan y Eita, 2007).

En el caso del Perú, la relevancia de las exportaciones agrarias, en el desarrollo rural, se manifiesta en la participación de la mano de obra, éste sigue representando, en promedio, el 29,6\% del empleo total del país, es decir, uno de cada tres peruanos es empleado o participa en el sector agrario. De acuerdo con el Ministerio de Agricultura y Riego del Perú (MINAGRI, 2016), las 
exportaciones del sector agrario representan el 12,8\% en el 2014 de las exportaciones del Perú. En consecuencia, es preciso invertir más en la agricultura y en los sistemas agroalimentarios, así como en la investigación y el desarrollo, para potenciar la productividad agraria e impulsar la innovación en agricultura sostenible, prosperidad rural y seguridad alimentaria (FAO, 2017).

El objetivo del presente estudio es determinar el efecto de la Ley de Promoción Agraria y la demanda mundial en las exportaciones agroalimentarios y alimentos procesados peruanos durante el periodo del 2001-2018.

\section{MATERIALES Y MÉTODOS}

\section{Variables}

Para este estudio fueron las exportaciones los países de la CAN (Bolivia, Colombia, Ecuador y Perú) y la importación mundial del grupo productos agroalimentarios y alimentos procesados. Se escogió a la CAN, por ser una organización creada en 1969, con el objetivo de formar un mercado común, teniendo políticas comunes de carácter social, económico y otros, entre los miembros (CAN, 2016). Se definió las variables como: Importaciones alimentos procesados y productos agroalimentarios mundo (I_ Mundo), Exportación alimentos procesados y productos agroalimentarios Perú ( $\mathrm{X}_{-}$ Perú), Exportación alimentos procesados y productos agroalimentarios Bolivia ( $\mathrm{X}_{-}$Bolivia), Exportación alimentos procesados y productos agroalimentarios Ecuador ( $\mathrm{X}_{-}$Ecuador). Exportación alimentos procesados y productos agroalimentarios Colombia (X_Colombia).

\section{Pruebas}

Estas son la causalidad de Granger y la cointegración de Johansen son técnicas econométricas usadas en esta investigación. La causalidad de Granger permitió el análisis bidireccional del agro - exportaciones y el crecimiento económico en Namibia (Jordaan y Eita, 2007). Asimismo, las pruebas de cointegración de Johansen y causalidad de Granger, en conjunto, demostraron que las exportaciones industriales principalmente $\mathrm{y}$, en menor medida, las exportaciones primarias causan impactos positivos en el producto neto de exportaciones en Boyacá, Colombia (Cáceres et al., 2018). Basado en las experiencias en mención se realizó la prueba de casualidad Granger, con el objetivo de determinar la causalidad entre las variables en estudio y la prueba cointegración de Johansen, para analizar la relación a largo plazo de las exportaciones del grupo de alimentos procesados y productos agroalimentarios de los miembros de CAN con las importaciones alimentos procesados y productos agroalimentarios mundo, los resultados se analizó entre los países con ley de promoción o fomento agraria (Perú y Ecuador), los países sin una real ley de promoción agraria (Colombia y ecuador) y el nivel de apertura la comercio internacional según los tratados internacionales vigentes.

\section{Data}

La información se obtuvo de International Trade Centre (ITC, 2019) de las exportaciones de cada miembro de la CAN e importación mundial dentro del grupo de alimentos procesados y productos agroalimentarios respectivamente. El deflactor del dólar de Estados Unidos se obtuvo del World Bank (WB, 2019). Los resultados de las tablas econométricas se empleó R Studio.

\section{Procedimiento}

Las variables se convirtieron en logaritmos neperianos, lo que permitió crear un modelo lineal. Con el fin de hallar la estacionariedad o noestacionariedad, se ejecutó la prueba de DickeyFuller, con los siguientes tipos: sin intercepto y sin tendencia (noconstant regress), con intercepto (regress) y con intercepto y tendencia (trend regress), de no encontrarse estacionariedad se llevó a la diferencia conveniente. Asimismo, se encontró los lags de cada variable de exportación. Finalmente, se determinó la relación a largo plazo y la causalidad entre las exportaciones de alimentos procesados y productos agroalimentarios de los países CAN e importaciones alimentos procesados y productos agroalimentarios mundo, aplicando la prueba de cointegración de Johansen y prueba de causalidad de Granger.

La relación de las variables en la vida real no es instantánea, sino que demanda un lapso de tiempo 
denominado lag (Gujarati y Porter, 2008). Usualmente, el número de lags es determinado por la frecuencia de la data (como también el tamaño de la muestra). En la data anual, uno o dos lags es suficiente (Wooldridge, 2012). Se consideró los criterios de información: la predicción final del error (FPE), el criterio de la información de Akaike (AIC), el criterio de información bayesiano de Schwars (SBIC o BIC), y el criterio de información de Hannan-Quinn (HQIC o HQC). De acuerdo, al estudio de Liew et al. (2008) muestra que SIC, FPE, HQC y BIC poseen un buen procedimiento escogiendo el verdadero orden del lags, inclusive si la muestra es pequeña, donde AIC sobrestima el verdadero valor de la probabilidad en más de dos tercios. En este trabajo se optó los criterios FPE y HQIC, según Liew et al. (2008).

La prueba de cointegración evalúa los términos de equilibrio a largo plazo entre variables no estacionarias, pero tiene una combinación lineal fija. En este estudio, se aplicó la prueba de Johansen donde se necesita que las variables sean estacionarias en el mismo grado (Syahril. et al., 2019). La metodología de Johansen usa el máximum estadístico y trace estadístico para determinar si las variables están cointegradas y el número de vectores cointegrados (Enders, 2015). La ecuación elemental que captura la prueba de Johansen es la siguiente:

$$
Z_{t}=A Z_{t-1}+\cdots+A_{n} Z_{t-n}+B x_{t}+\epsilon_{t}(1)
$$

En esta ecuación (1) $Z_{t}$, es el vector para las variables independientes y dependientes, $\mathrm{x} \_\mathrm{t}$ es la variable no aleatoria, $\epsilon_{-} \mathrm{t}$ es el término de error de corrección, para todo: t es el tiempo y n el número de vectores (Naidu et al., 2017).

Granger comienza desde la preposición de que el presente y el pasado no pueden causar el futuro. Si la causalidad se define en términos de previsibilidad, por lo tanto, la variable $\mathrm{X}$ causa variable $\mathrm{Y}$. Si el presente $\mathrm{Y}$ puede predecirse mejor utilizando valores pasados de $\mathrm{X}$ (rezagados) que no al hacerlo, con respecto a un conjunto de información dado que incluye X e Y (Al Barghouthi et al., 2019).
El test de Granger está definida por dos variables, $\mathrm{X}$ ecuación (2) e Y ecuación (3), escritas:

$$
\begin{aligned}
& X_{t}=\sum_{j=1}^{p} \alpha_{j} X_{t-j}+\sum_{j=1}^{p} \beta_{j} \mathrm{Y}_{t-j}+\mu_{t}(2) \\
& \mathrm{Y}_{t}=\sum_{j=1}^{p} \eta_{j} X_{t-j}+\sum_{j=1}^{p} \gamma_{j} \mathrm{Y}_{t-j}+v_{t}(3)
\end{aligned}
$$

Las hipótesis nulas son testeadas:

$$
H_{1}: \eta_{j}=0, j=1 \ldots p(4)
$$

Lo que significa que las $\mathrm{X}$ no son una causa de Granger de Y.

$$
H_{1}: \beta_{j}=0, j=1 \ldots p,(5)
$$

Nos indica que las $\mathrm{Y}$ no son una causalidad de Granger en las X. Si ninguna de las hipótesis (4 y 5) es rechazada lo que significa que las $\mathrm{X}$ no son causalidad Granger de las Y, y viceversa. Si la primera hipótesis (4) es rechazada, significa que las $\mathrm{X}$ causalidad de Granger las Y. El rechazo de la segunda hipótesis (5) demuestra que las Y causan las X. Si todas las hipótesis son rechazadas esto significa la doble dirección de la causalidad entre las X y las Y (Jordaan y Eita, 2007).

\section{RESULTADOS Y DISCUSIÓN}

\section{Estacionariedad (Prueba de Dickey-Fuller)}

Al aplicar la Prueba de Dickey-Fuller, se observó no presencia de no Estacionariedad (|Test Statistic| < Critical Value) en todas las variables (Tabla 1). Al someterse en su primera diferencia las variables resultaron estacionales.

\section{Lags (rezagado)}

Se determinó lags a las exportaciones de alimentos procesados y productos agroalimentarios para Perú, Bolivia, Ecuador y Colombia, obteniendo los siguientes resultados, de acuerdo al Tabla 2, aplicando el menor valor del criterio(s) FPE - HQIC, según Liew et al. (2008). Los países Perú, Bolivia, Colombia y Ecuador, obtuvieron de lag (2) o un lapso de dos periodos de tiempo. 
Tabla 1. Estacionariedad de Alimentos procesados y productos agroalimentarios de las importaciones del mundo y exportaciones de Perú, Bolivia, Ecuador y Colombia.

\begin{tabular}{|c|c|c|c|c|c|c|c|c|c|}
\hline \multicolumn{10}{|c|}{ Augmented Dickey-Fuller Test Unit Root Test } \\
\hline Variable/ Tipo & \multicolumn{3}{|c|}{$\begin{array}{c}\text { Critical Value } \\
\text { Porcentaje }\end{array}$} & 点 & 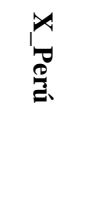 & 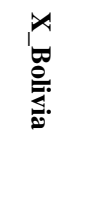 & 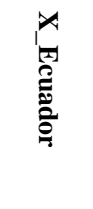 & $\begin{array}{l}x \\
0 \\
0 \\
0 \\
0 \\
0\end{array}$ & 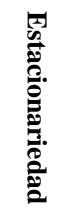 \\
\hline \multicolumn{10}{|c|}{ Logaritmo Natural } \\
\hline Regress & -3.75 & $-3,00$ & $-2,63$ & $-1,38$ & $-1,38$ & $-1,46$ & $-1,93$ & $-0,58$ & $\mathrm{NO}$ \\
\hline Trend regress & $-4,38$ & $-3,60$ & $-3,24$ & $-1,36$ & $-1,36$ & $-1,36$ & $-2,00$ & $-2,55$ & NO \\
\hline Noconstant regress & $-2,66$ & $-1,95$ & $-1,60$ & 1,93 & 1,93 & 1,08 & 1,09 & 2,37 & $\mathrm{NO}$ \\
\hline \multicolumn{10}{|c|}{ Primera Diferencia $(\Delta)$} \\
\hline Regress & $-3,75$ & $-3,00$ & $-2,63$ & $-3,88$ & $-3,88$ & $-3,83$ & $-4,62$ & $-4,19$ & SI \\
\hline Trend regress & $-4,38$ & $-3,60$ & $-3,24$ & $-4,10$ & $-4,10$ & $-3,89$ & $-4,89$ & $-4,02$ & SI \\
\hline Noconstant regress & $-2,66$ & $-1,95$ & $-1,60$ & $-3,21$ & $-3,21$ & $-3,71$ & $-4,31$ & $-3,20$ & SI \\
\hline
\end{tabular}

Tabla 2. Determinación del número de Lags - Criterios de Información.

\begin{tabular}{lrrrr}
\hline $\begin{array}{l}\text { Criterios de información } \\
\text { (País) }\end{array}$ & Perú & Bolivia & Colombia & Ecuador \\
\hline Lag & 2 & 2 & 2 & 2 \\
FPE &, 00001 &, 000098 &, 000034 & 0,000097 \\
AIC & $-6,53907$ & $-4,27633$ & $-5,33601$ & $-3,64872$ \\
HQ & $-7,49413$ & $-5,23139$ & $-6,29106$ & $-3,73805$ \\
SC & $-6,61634$ & $-4,3536$ & $-5,41328$ & $-3,21415$ \\
\hline
\end{tabular}

\section{Causalidad de Granger}

Se realizaron con lags (2), para todas las variables. La prueba de causalidad mostró si la demanda mundial de alimentos (mundo) explica o causa la exportación de alimentos procesados y productos agroalimentarios y viceversa. En los casos de Perú y el Mundo, Bolivia y el Mundo, Ecuador y el Mundo e Colombia y el Mundo (Tabla 3).

Las Variables Excluidas de Perú, Bolivia, Colombia y Perú en relación con la variable Mundo tienen una probabilidad ( $\mathrm{pr}(>\mathrm{F}))$ mayor que $5 \%$; por lo tanto, no se puede rechazar la hipótesis nula con un lags (2) lo que significa que la exportación alimentos procesados y productos agroalimentarios de Perú, Bolivia, Colombia y Perú en cada caso en particular no causan o inciden la demanda mundial de alimentos. En caso opuesto (variable excluida es el Mundo en relación al Perú, Bolivia, Colombia y
Ecuador) presenta una probabilidad mayor al 5\% a un lags (2) lo que se traduce que la demanda mundial de alimentos no explica o causan exportación alimentos procesados y productos agroalimentarios de Perú, Bolivia, Colombia y Perú en cada caso en individualmente.

\section{Cointegración (Prueba de Johansen)}

En los casos de Perú y Ecuador de la Tabla 4, se rechaza al 5\% de significancia la hipótesis nula de cointegración, pero no se rechaza la relación de cointegración por lo tanto se puede concluir que existió una relación de largo plazo. En los casos de Colombia y Bolivia se acepta al 5\% de significancia la hipótesis nula de cointegración, por lo tanto, no existió ninguna relación entre las variables. En los casos de Perú y Ecuador existió una relación de largo plazo entre variables, interpretándose que en el largo plazo la demanda de alimentos (importación de 
alimentos procesados y productos agroalimentarios mundo) incidió en la exportación de alimentos procesados y productos agroalimentarios de Perú y Ecuador

Tabla 3. Prueba de Causalidad de Granger entre el Mundo y los países de Perú, Bolivia, Colombia y Ecuador.

\begin{tabular}{|c|c|}
\hline$\Delta$ X_Peru, $\Delta$ I_Mundo & Res.Df Df \\
\hline Model 1: $\Delta$ I_Mundo $\sim \operatorname{Lags}\left(\Delta \_I \_M u n d o, 1: 2\right)+\operatorname{Lags}\left(\Delta \_X \_P e r u, 1: 2\right)$ & 110 \\
\hline Model 2: $\Delta$ _I_Mundo $\sim$ Lags $\left(\Delta \_I \_M u n d o, 1: 2\right)$ & $2 \quad 12-21,2535 \underline{0,3268}$ \\
\hline \multicolumn{2}{|l|}{$\Delta \mathrm{I} \_$Mundo, $\Delta \mathrm{X} \_$Peru } \\
\hline Model 1: $\Delta \_X \_P e r u \sim \operatorname{Lags}\left(\Delta \_X \_P e r u, 1: 2\right)+\operatorname{Lags}\left(\Delta \_I \_M u n d o, 1: 2\right)$ & $1 \quad 10$ \\
\hline Model 2: $\Delta$ _X_Peru $\sim$ Lags $\left(\Delta \_X \_P e r u, 1: 2\right)$ & $2 \quad 12-21,4595 \underline{0,2779}$ \\
\hline \multicolumn{2}{|l|}{$\Delta \mathrm{X}$ B Bolivia, $\Delta \mathrm{I}$ I Mundo } \\
\hline Model 1: $\Delta$ _I_Mundo $\sim \operatorname{Lags}\left(\Delta \_I \_M u n d o, 1: 2\right)+\operatorname{Lags}\left(\Delta \_\right.$X_Bolivia, 1:2) & 110 \\
\hline Model 2: $\Delta$ _I_Mundo $\sim$ Lags $\left(\Delta \_I \_M u n d o, 1: 2\right)$ & $2 \quad 12-20,3566 \underline{0,7086}$ \\
\hline \multicolumn{2}{|l|}{$\Delta \mathrm{I} \_$Mundo, $\Delta \mathrm{X} \_$Bolivia } \\
\hline Model 1: $\Delta$ _X_Bolivia $\sim \operatorname{Lags}\left(\Delta \_X \_B o l i v i a, 1: 2\right)+\operatorname{Lags}(\Delta$ _I_Mundo, 1:2) & 10 \\
\hline Model 2: $\Delta \_X \_B o l i v i a ~ \sim \operatorname{Lags}\left(\Delta \_X \_B o l i v i a, 1: 2\right)$ & $2 \quad 12-21,5048 \underline{0,2683}$ \\
\hline \multicolumn{2}{|l|}{$\Delta \bar{X} \_$Ecuador, $\Delta$ I_Mundo } \\
\hline Model 1: $\Delta$ _I_Mundo $\sim \operatorname{Lags}\left(\Delta \_I \_M u n d o, 1: 2\right)+\operatorname{Lags}\left(\Delta \_X \_E c u a d o r, 1: 2\right)$ & 110 \\
\hline Model 2: $\Delta$ I I Mundo $\sim \operatorname{Lags}(\Delta$ I Mundo, 1:2) & $2 \quad 12-20,9045 \underline{0,4354}$ \\
\hline \multicolumn{2}{|l|}{$\Delta \mathrm{I} \_$Mundo, $\Delta \mathrm{X} \_$Ecuador } \\
\hline 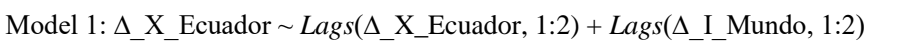 & 10 \\
\hline Model 2: $\Delta \_X \_E c u a d o r ~ \sim \operatorname{Lags}\left(\Delta \_X \_E c u a d o r, 1: 2\right)$ & $2 \quad 12-23,674 \underline{0,06364}$ \\
\hline \multicolumn{2}{|l|}{$\Delta \mathrm{X} \_$Colombia, $\Delta \mathrm{I}$ Mundo } \\
\hline Model 1: $\Delta$ _I_Mundo $\sim \operatorname{Lags}\left(\Delta \_I \_M u n d o, 1: 2\right)+\operatorname{Lags}\left(\Delta \_X \_C o l o m b i a, 1: 2\right)$ & 10 \\
\hline Model 2: $\Delta$ I Mundo $\sim \operatorname{Lags}(\Delta$ I Mundo, 1:2) & $2 \quad 12-2 \quad 1,0403 \underline{0,3887}$ \\
\hline \multicolumn{2}{|l|}{$\Delta \mathrm{I} \_$Mundo, $\Delta \mathrm{X} \_$Colombia } \\
\hline Model 1: $\Delta$ _X_Colombia $\sim \operatorname{Lags}\left(\Delta \_X \_C o l o m b i a, 1: 2\right)+\operatorname{Lags}\left(\Delta \_I \_M u n d o, 1: 2\right)$ & 10 \\
\hline Model 2: $\Delta \_X \_C o l o m b i a ~ \sim \operatorname{Lags}\left(\Delta \_X \_\right.$Colombia, $\left.1: 2\right)$ & $2 \quad 12-20,1195 \underline{0,8886}$ \\
\hline
\end{tabular}

Tabla 4. Prueba de Cointegración entre Mundo y los países de Perú, Bolivia, Colombia y Ecuador.

\begin{tabular}{lccccc}
\hline $\begin{array}{l}\text { Critical Value } \\
\text { maximun }\end{array}$ & $\mathbf{5 \%}$ & Perú & Bolivia & $\begin{array}{c}\text { Mundo } \\
\text { Colombia }\end{array}$ & Ecuador \\
\hline $\mathrm{r}=0$ & 15,41 & 35,2297 & $\underline{10,67}$ & $\underline{10,5605}$ & 20,2925 \\
$\mathrm{r}<=1$ & 3,76 & $\underline{3,1699}$ & 1,5544 & 2,6403 & $\underline{0,9551}$ \\
eigenvalue & & 0,91509 & 0,50401 & 0,45624 & 0,77406 \\
\hline
\end{tabular}

Ley $\mathrm{N}^{\circ} 54$ - Ley de Desarrollo Agrario, tiene por objeto el fomento, desarrollo y protección integrales del sector agrario en Ecuador, codificado por el congreso en 1997 (FAO, 1997) y la Ley 27360 promulgada con el objetivo de priorizar las inversiones y desarrollo agrario en el Perú, constituyen las dos (02) legislaciones que promueven el desarrollo agrario dentro de los países de la CAN. Ley general de desarrollo agropecuario y pesquero de Colombia (Ley 101 de 1993) según el artículo 64, se refiere al acceso progresivo de los trabajadores agrarios a la propiedad de la tierra y a los servicios y el artículo 65, menciona la protección gubernamental de la producción local (Leibovich, 1994), lo indica que es una ley cuya prioridad es la redistribución de la tierra y protección al sector agropecuario y pesquero. En Bolivia, la ley INRA o Ley de la Segunda Reforma Agraria establece la necesidad de garantizar el acceso con equidad al recurso tierra, otorgar seguridad jurídica a los 
poseedores de esta y promover el uso sostenible del recurso (Ruiz, 2017), en realidad la ley INRA no constituyó una ley de promoción o fomento al sector. Sin embargo, Bolivia exportó el $88 \%$ del total en el sector a los miembros de la Comunidad Andina se presume que beneficio del tratado de comercio comunitario (Anexo 4). Perú y Colombia son los países con mayores tratados de libre comercio seguidos por Ecuador. De acuerdo con Díaz (2017) sobre Colombia, manifiesta: el desarrollo de acuerdos bilaterales como el Tratado de Libre Comercio (TLC) no genera a largo plazo resultados favorables para algunos productos como el arroz y el maíz...A Colombia le falta mucha competitividad para enfrentarse al Tratado del Libre Comercio con Estados Unidos. Además, según información del ITC (2020) se aprecia en Anexo 3, el crecimiento de las exportaciones del sector, en el caso de las exportaciones colombianas es casi plano en comparación de las exportaciones Perú y Ecuador, que son crecientes en grupo de exportaciones alimentos procesados y agroalimentarios.

\section{CONCLUSIÓN}

La prueba de Causalidad de Granger demostró que la importación mundial de alimentos procesados y agroalimentarios no causa las exportaciones de alimentos procesados y agroalimentarios de los países de la comunidad andina, y viceversa. La prueba de Cointegración de Johansen mostró que existe integración o relación de largo plazo para las exportaciones alimentos procesados y agroalimentarios de Perú y Ecuador con la importación mundial de alimentos procesados y agroalimentarios, coincidentemente los dos países con leyes de promoción al sector agropecuario y economías abiertas al mercado mundial.

En el caso de Colombia a pesar de tener una serie de tratados de libre comercio, se presume que no ha aprovechado la demanda de alimentos mundial en el sector. Bolivia aparentemente se ha favorecido del tratado comercial dentro de la comunidad andina en el sector, siendo una economía sin mayores tratados de libre comercio con el mundo. Perú y Ecuador, con leyes de promoción agropecuaria y economías con tratados de libre comercio, se beneficiaron de la demanda de alimentos mundial a diferencia de Bolivia y Colombia, entonces se puede afirmar que las exportaciones de alimentos procesados y agroalimentarios en el caso de Perú se beneficiaron de la demanda de alimentos mundial, gracias a la implementación de la Ley 27360.

\section{Conflictos de intereses}

Los autores firmantes del presente trabajo de investigación declaran no tener ningún potencial conflicto de interés personal o económico con otras personas $\mathrm{u}$ organizaciones que puedan influir indebidamente con el presente manuscrito.

\section{Contribuciones de los autores}

Preparación y ejecución: CC, LG; Desarrollo de la metodología: CC, LG; Concepción y diseño: CC, LG; Edición del artículo: CC, LG; Supervisión del estudio: CC, LG.

\section{LITERATURA CITADA}

- Al Barghouthi, S., Ijaz, U., \& Ghaida, R. (2019). Testing the Efficiency of ASE by the Two Step Regression Based Technique, the Johansen Multivariate Technique Cointegration, and Granger Causality. Electronic Journal of Applied Statistical Analysis [Online], 9.3 (2016): $572-586$.

- Cáceres, W., Agudelo, C., \& Tejedor, E. (2018). Las exportaciones y el crecimiento económico en Boyacá Colombia 1980-2015. Apuntes del Cenes, 37(65), 175-211. Disponible en: https://doi.org/10.19053/01203053.v37.n65.201 8.7122

- Comunidad Andina Naciones. [CAN]. (2016). Dimensión Económica Social de la Comunidad Andina. Secretaría General de la Comunidad Andina. Lima- Perú.1-80:51 p.

- Díaz, V. (2017). Incidencia del Tratado de Libre Comercio con Estados Unidos en La Competitividad del Sector Agrícola Colombiano: Casos Del Arroz y Maíz. Revista de Economía del Caribe, (19), 154-182. 
- El Peruano. (2000). El Congreso de la República: Ley Número 27360.Lima, martes 31 de octubre del 2000: 194515.

- Enders, W. (2015). Applied Econometric Time Series. University of Alabama. - Fourth edition.1-419: $400 \mathrm{p}$.

- Gujarati, D., \& Porter, D. (2008). Basic Econometrics. McGraw-Hill Education; 5 edition $.618 \mathrm{p}$.

- International Trade Centre. [ITC]. (2019). Exportaciones (Perú, Bolivia, Colombia y Ecuador) e Importaciones (Mundo). Processed food and agro-based products. Consultado el 14 octubre 2019. Disponible en: http://www.intracen.org/itc/market-infotools/trade-statistics/.

- Jordaan, A. C., \& Eita, J.H. (2007). Export and Economic Growth in Namibia: A Granger Causality Analysis. South African Journal of Economics, $\quad 75, \quad 540-547$. https://doi.org/10.1111/j.18136982.2007.00132.x

- La Organización de las Naciones Unidas para la Agricultura y la Alimentación. [FAO]. (1997). Ley $\mathrm{N}^{\mathrm{o}} 54$ - Ley de Desarrollo Agrario (Ecuador). Consultado el 2 noviembre 2019. Disponible en: http://www.fao.org/faolex/results/details/en/c/L EX-FAOC004813

- La Organización de las Naciones Unidas para la Agricultura y la Alimentación. [FAO]. (2017). El futuro de la alimentación y la agricultura: Tendencias y desafíos - versión resumida. 1 - 52: 47 p. Disponible en: http: www.fao.org/publications.

- La Superintendencia Nacional de Aduanas y de Administración Tributaria. [SUNAT]. (2019). Principales Empresas por Sectores Económicos, Principales 500 Productos No Tradicionales. Consultado el 12 noviembre 2019. Disponible en: http://www.sunat.gob.pe/ estadcomExt/modelo_web/.

- Leibovich, J. (1994). Comentarios a la ley general de desarrollo agropecuario y pesquero (Ley 101 de 1993) Colombia. Bogotá Colombia. Coyuntura Económica. 24(4), 165169. Fedesarrollo, Bogotá - Colombia.
- León, L. (2013). Acuerdos Comerciales del Perú. Congreso de la República del Perú: 17-21: 17 p.

- LEY No 27360. Ley que aprueba las Normas de Promoción del Sector Agrario Disponible en https://www2.congreso.gob.pe/sicr/cendocbib/c on4_uibd.nsf/5C947E120537341B05257B7A00 4B13E5/\$FILE/27360.pdf

- Liew, V., Shitan, M., Chee, K., \& Chee, W. (2008). Performance of Autoregressive Order Selection Criteria: A Simulation Study. University Putra Malaysia Press J. Sci. \& Technol. 16 (2): 171 - 176: 175.

- Ministerio de Agricultura y Riego del Perú. [MINAGRI]. (2016). Política Nacional Agraria. Hecho el Depósito Legal en la Biblioteca Nacional del Perú No 2016 - 05292.1-42: 10p.

- Naidu, S., Pandaram, A., \& Chand, A. (2017). A Johansen Co-integration Test for the Relationship between Remittances and Economic Growth of Japan. Published by Canadian Center of Science and Education. Modern Applied Science, 11(10), 137-151. https://doi.org/10.5539/mas.v11n10p137

- Organización de las Naciones Unidas para la Agricultura y la Alimentación y Corporación Andina de Fomento. [FAO - CAF]. (2019). PERÚ NOTA DE ANÁLISIS SECTORIAL: Agricultura y Desarrollo Rural.1-65: VII.

- Organización Mundial de Comercio. [OMC]. (2019). Perú y la OMC. Consultado el 1 octubre 2019.Disponible en: Disponible en: https://www.wto.org/spanish/thewto_s/countrie s_s/peru_s.htm

- Ruiz, A. (2017). Reformas agrarias y sus rastros en ruralidades contemporáneas: los casos de Bolivia y Chile. Valdivia, Chile. Revista Austral de Ciencias Sociales, 32, 99-119.

- Sistema de Información de Comercio Exterior. [SICE]. (2019). Acuerdos Comerciales de Perú, Bolivia, Colombia y Ecuador. Organización de los Estados Americanos, Consultado el 2 noviembre 2019. Disponible en: http://www.sice.oas.org/default_s.asp.

- Sociedad de Comercio Exterior del Perú [COMEX Perú]. (2019). Ley 27360 - Ley de promoción del sector agrario: 3, 9-10 p.

- Syahril, R.M., Sofyan, S., Shabri, A., Zulham, T., Jumadil, S., Saiful, B., \& Irmayani. (2019). 
The Effect of Global Prices of Crude Palm Oil, Marketing Margins and Palm Oil Plantations on the Environmental Destruction: An Application of Johansen Co-integration Approach. International Journal of Energy Economics and Policy, 9(4), 305-312.

- Wooldridge, J. (2012). Introductory Econometrics: A Modern Approach. Michigan
State University. Cengage Learning.1-910: 642 p.

- World Bank. [WB]. (2019). Deflactor del dólar de Estados Unidos. Consultado el 1 octubre $2019 . \quad$ Disponible en: https://data.worldbank.org/indicator/NY.GDP.D EFL.ZS 